\title{
Coronary sinus lead delay index for optimization of coronary sinus lead placement
}

\author{
Mevlüt Koç MD | Onur Kaypakli MD (D) | Gökhan Gözübüyük MD | \\ Durmus Yıldıray Şahin MD
}

Department of Cardiology, Adana Health Practices and Research Center, Health

Sciences University, Adana, Turkey

\section{Correspondence}

Onur Kaypakli, Department of Cardiology, Adana Health Practices and Research Center, Health Sciences University, Adana, Turkey.

Email: onurkaypakli@hotmail.com
Aim: Optimization of coronary sinus (CS) lead position to the latest activated left ventricular (LV) area is important to increase cardiac resynchronization therapy (CRT) response. We aimed to detect the relationship between coronary sinus lead delay index (CSDI) and echocardiographic, electrocardiographic response to CRT treatment.

Methods: We prospectively included 137 consecutive patients with heart failure (HF) diagnosis, QRS $\geq 120 \mathrm{~ms}$, left bundle branch block (LBBB), New York Heart Association score (NYHA) II-IV, LV ejection fraction (LVEF) $<35 \%$ and scheduled for CRT (84 male, 53 female; mean age $65.1 \pm 10.1$ years). Echocardiographic CRT response was defined as $\geq 15 \%$ reduction in LV end-systolic volume (LVESV). CS lead sensing delay was calculated as the time interval from the onset of surface QRS wave to the onset of depolarization wave recorded from the CS lead by using the CS pacing lead as a bipolar electrode. CSDI was calculated by dividing the CS lead sensing delay by the QRS duration.

Results: LVESV reduction was associated with baseline QRS width ( $r=.257, p=.002)$, QRS narrowing $(r=.396, p<.001)$, CSDI $(r=.357, p<.001)$, and NT-proBNP $(r=-0.213, p=.022)$ in bivariate analysis. In logistic regression analysis, CSDI was found to be only independent parameter for predicting significant LVESV reduction (Beta $=0.318, p<.001$ ). CSDI was also found to be significantly associated with LVEF increase $(r=.244, p=.004)$ and QRS narrowing $(r=.178, p=.046)$.

Conclusion: CSDI may be used as a marker to predict the favorable response to CRT. It may be useful to integrate CSDI to CRT implantation procedure in order to minimize nonresponders.

KEYWORDS

coronary sinus lead delay index, CRT response, QRS width

\section{1 | INTRODUCTION}

Left ventricular (LV) dysfunction because of atrioventricular, interventricular and intraventricular conduction delays were intended to be corrected with cardiac stimulation with cardiac resynchronization therapy (CRT). CRT improves LV mechanical function by reducing dyssynchrony between the interventricular septum and LV lateral, posterolateral wall. CRT devices have been shown to improve heart failure (HF) symptoms, quality of life, HF hospitalizations and mortality in studies. However, a significant proportion of apparently suitable patients do not get any detectable benefit (Daubert et al., 2012).

It is not clear what the main factors of response to CRT are. But, better patient selection is not enough to reduce non responders. Because preimplantation factors are not the only predictors of CRT response. Factors associated with implantation and postimplantation period are also as important as preimplantation factors. In order to 
reduce nonresponders to CRT treatment, optimization of LV lead position is an important field of research. Coronary sinus (CS) branch adjacent to the latest activated LV area has been shown to be the optimal site for CS lead placement (Khan et al., 2012; Kydd et al., 2014; Ypenburg et al., 2008). Different echocardiographic and electroanatomic mapping (EAM) methods were studied to determine the most favorable coronary sinus branch adjacent to the latest activated LV area (Del Greco, Marini, \& Bonmassari, 2012; Khan et al., 2012; Liang et al., 2012a,b; Ypenburg et al., 2008).

In our study, we aimed to detect the relationship between coronary sinus lead delay index (CSDI) and echocardiographic, electrocardiographic response to CRT treatment.

\section{2 | METHODS}

\section{1 | Study protocol and study population}

We prospectively included 137 consecutive patients with HF diagnosis, QRS $\geq 120$ ms, LBBB, NYHA II-IV, LVEF < 35\% and scheduled for CRT (84 male, 53 female; mean age $65.1 \pm 10.1$ years). Symptomatic patients despite optimum medical treatment scheduled for CRT implantation with LV ejection fraction (LVEF) less than 35\% were included to the study. New York Heart Association (NYHA) scores of patients were determined. Patients with advanced renal insufficiency (serum creatinine $>2.0 \mathrm{mg} / \mathrm{dl}$ ), hypothyroidism, hyperthyroidism, respiratory diseases (COPD, chronic bronchitis, pulmonary embolism), primary pulmonary hypertension, acute coronary syndrome in the last 2 months, isolated right HF, congenital heart disease, severe valve disease (aortic stenosis, mitral stenosis, and regurgitation) were excluded from the study. The Local Ethics Committee approved the study protocol, and each participant provided written informed consent.

After assessment of detailed medical history and a complete physical examination, the baseline characteristics of patients including age, gender, hypertension, diabetes, hyperlipidemia, current smoking status, family history of cardiac disease and medications were recorded for all patients. ECG, telecardiography, complete blood count, fasting blood glucose, uric acid, NT-proBNP, serum electrolytes, serum lipids, renal and liver function tests were performed. All patients had QRS duration greater than $120 \mathrm{msn}$. Patients were classified according to the NYHA staging system by two cardiologists, if there is no consensus, NYHA class was determined by joint decision taken by a third cardiologist. Patients' medical treatment was adjusted according to recent HF guidelines and their clinical stage.

\subsection{Echocardiographic and electrocardiographic assessment}

Echocardiographic assessment was made by using a $2.5-3.5 \mathrm{MHz}$ transducer (Philips HD11 ultrasound system, Bothell, WA, USA) with parasternal long and short axis, apical two and four chamber views. Baseline transthoracic echocardiography measurements were performed before CRT implantation. Echocardiographic measurements were made according to the suggestions of the American Society of
Echocardiography (Sahn, De Maria, Kisslo, \& Weyman, 1978). LV transverse axis dimensions at end-diastole, LV end-diastolic dimension (onset of the $\mathrm{Q}$ wave of the electrocardiogram), and LV end-systolic dimension were measured from $\mathrm{M}$-mode recordings according to recommendations of the American Society of Echocardiography (Sahn et al., 1978). This was obtained from the parasternal long-axis view with the M-mode cursor positioned adjacent to the tips of the mitral valve leaflets, using leading-edge methods. LV end-systolic volume (LVESV), LV end-diastolic volume, and LVEF were assessed using Simpson's equation using the apical 4-chamber view (Schiller, 1991). PW Doppler, color Doppler, PW and color tissue Doppler evaluation were performed respectively. Echocardiographic wall motion anomalies, wall thickness, heart valves, left and right heart chambers were assessed. Intraventricular mechanical delay and dyssynchrony were measured from parasternal long-axis M-mode recordings and from TDI and color TDI recordings obtained with the cursor positioned at the basal lateral and septal part of the LV wall that closest to mitral annulus. We calculated the delay between the septal and posterior wall in parasternal long-axis M-mode recordings for intraventricular dyssynchrony (Rickard, 2012). In TDI we calculated time interval from the onset of QRS and the beginning of systolic velocity. Septal-lateral delay for the beginning of systolic velocity were recorded for intraventricular dyssynchrony (Rickard, 2012). Same echocardiographic measurements were recorded after at least 3 months. Echocardiographic CRT response was defined as $\geq 15 \%$ reduction in LVESV. QRS duration was measured in the lead with widest QRS complex. QRS duration was recorded before CRT implantation and after at least 3 months. The difference between baseline and control QRS duration was recorded as QRS narrowing.

\subsection{Pacemaker implantation and measures}

CRT device implantations were performed transvenously targeting a lateral or posterolateral vein in the coronary sinus for the LV lead position. There has been no need for surgical placement of epicardial lead because all LV leads were placed successfully with transvenous approach. Bipolar leads which can pace from two regions were used for LV leads. All CRT devices in this study had defibrillator function. CRT devices were commonly programmed with an atrioventricular sensed delay of $100 \mathrm{~ms}$ and paced delay of $130 \mathrm{~ms}$, with optimization performed according to standard institutional protocols. Shock and treatment zones for ventricular tachycardia were set at the end of the process. Medications were recorded immediately before implantation of the CRT device with subsequent titration of medications made at the discretion of patients' outpatient physicians. Pacemaker measurements was made with MEDTRONiC Programmer 2090 programmable pacemaker analyzer. During the implantation; the $\mathrm{P}$ wave and R-wave amplitude, electrode impedance, threshold values of LV, RV and RA leads were calculated. Baseline value of pacing threshold was defined as the lowest voltage which can form five consecutive stimuli with 0.4 pulse width. Basal impedance was recorded as $400-1000$ ohms $(\Omega)$, R wave as $>5 \mathrm{mV}$, the $\mathrm{P}$ wave as $>2 \mathrm{mV}$. During the implantation; CS lead sensing delay was calculated as the time interval from the onset of surface QRS wave to the onset of depolarization wave recorded from the CS 
TABLE 1 Comparison of the baseline clinical and demographic features

\begin{tabular}{|c|c|c|c|}
\hline & $\begin{array}{l}\text { Nonresponders (LVESV } \\
\text { reduction < 15\%) } \\
n=67\end{array}$ & $\begin{array}{l}\text { Responders (LVESV } \\
\text { reduction } \geq 15 \% \text { ) } \\
n=70\end{array}$ & $p$ \\
\hline Age (years) & $66.7 \pm 9.4$ & $63.6 \pm 10.4$ & .073 \\
\hline Gender (male, \%) & $40(59)$ & $44(62)$ & .705 \\
\hline $\begin{array}{l}\text { Etiology (ischemic/ } \\
\text { nonischemic) }\end{array}$ & $33 / 34$ & $29 / 41$ & .358 \\
\hline $\begin{array}{l}\text { Baseline NYHA (II/III/ } \\
\text { IV) }\end{array}$ & $18 / 42 / 7$ & $26 / 42 / 2$ & .124 \\
\hline $\mathrm{DM}(n, \%)$ & $23(34)$ & $28(40)$ & .492 \\
\hline $\mathrm{HT}(n, \%)$ & $42(62)$ & $36(51)$ & .183 \\
\hline $\begin{array}{l}\text { Body mass index }(\mathrm{kg} / \\
\left.\mathrm{m}^{2}\right)\end{array}$ & $26.2 \pm 3.0$ & $25.6 \pm 4.5$ & .397 \\
\hline Hyperlipidemia (n, \%) & $27(40)$ & $38(54)$ & .101 \\
\hline Smoking status ( $n, \%)$ & $8(11)$ & $8(11)$ & .926 \\
\hline ACE inhibitor ( $n, \%)$ & $42(63)$ & $39(56)$ & .399 \\
\hline $\begin{array}{l}\text { Angiotensin II blocker } \\
(n, \%)\end{array}$ & $17(25)$ & $14(20)$ & .450 \\
\hline Beta-blockers ( $n, \%)$ & 59 (89) & $63(91)$ & .707 \\
\hline Digoxin (n, \%) & $23(34)$ & $16(23)$ & .135 \\
\hline Furosemide (n, \%) & $61(92)$ & $62(89)$ & .600 \\
\hline Spironolactone (n, \%) & $51(77)$ & $47(68)$ & .233 \\
\hline Ivabradine (n, \%) & $12(18)$ & $10(14)$ & .587 \\
\hline Statin (n, \%) & $18(27)$ & $18(26)$ & .876 \\
\hline $\begin{array}{l}\text { Acetylsalicylic acid ( } n \text {, } \\
\%)\end{array}$ & $38(57)$ & $41(64)$ & .828 \\
\hline
\end{tabular}

NYHA, New York Heart Association; DM, diabetes mellitus; HT, hypertension; ACE, angiotensin converting enzyme. lead by using the CS pacing lead as a bipolar electrode. CSDI was defined with the formula of CSDI = CS lead sensing delay/QRS duration.

\section{4 | Blood sampling}

Venous blood samples were obtained following overnight fasting (8 hrs), after thirty-minute rest in the sitting position. Samples were taken from cubital vein into blood tubes and immediately stored on ice at $4^{\circ} \mathrm{C}$. Blood counts were measured by a Sysmex K-1000 (Block Scientific, Bohemia, NY, USA) autoanalyzer within 5 min of sampling. Plasma triglyceride, low-density lipoprotein (LDL), high-density lipoprotein (HDL), glucose, uric acid, NT-proBNP, liver function tests, serum electrolytes, and creatinine concentrations were measured with an automated chemistry analyzer (Abbott Aeroset, North Chicago, IL, USA) using commercial kits (Abbott).

\section{5 | Statistical analyses}

Statistical analyses were conducted using SPSS, version 14.0, (SPSS Inc. Chicago, IL, USA). Data are expressed as mean \pm SD for continuous variables and percentage for categorical variables. The ShapiroWilk test was used to test normality and a $p$ value $>.05$ was defined as normally distributed data. Continuous variables that showed normal distribution were compared using the Student $t$ test and ANOVA, whereas the Mann-Whitney $U$ test and Kruskal-Wallis test were used for nonnormally distributed samples. Categorical variables and frequencies were compared by means of the chi-square test. Statistical significance was defined as a $p$-value $<.05$ for all comparisons. Pearson's and Spearman's correlation were used to examine the relationship between continuous variables.

The factors associated with significant reduction in LVESV were tested by univariate and multivariate logistic regression analyses. Variables with a $p$-value $<0.05$ in the univariate analysis were tested in the multivariate model. Results were expressed as the $p$ value and hazard ratio (HR) in $\mathrm{Cl}$ of $95 \%$. ROC analysis was made to determine the cutoff value of CSDI to detect significant reduction in LVESV.

\section{3 | RESULTS}

\section{1 | Comparison of baseline characteristics in patients with and without significant $(\geq 15 \%)$ reduction in LVESV}

A comparison of the baseline characteristics is shown in Table 1. There were no significant differences between the groups. Use of cardiac drugs were found to be similar between the two groups. 


\subsection{Comparison of laboratory parameters in patients with and without significant $(\geq 15 \%)$ reduction in LVESV}

Laboratory parameters were similar in two groups. Hemoglobin levels were significantly higher, creatinine and NT-proBNP levels were significantly lower $(p<.05$, for all) in responder group (Table 2$)$.

\section{3 | Comparison of electrocardiographic and echocardiographic parameters in patients with and without significant $(\geq 15 \%)$ reduction in LVESV}

Baseline QRS width, QRS narrowing, and CSDI were found to be higher in responder group ( $p<.05$, for all). LVESV reduction and LVEF increase were found to be higher in responder group ( $p<.05$, for all). Other echocardiographic parameters were similar between the two groups (Table 3 ).

\section{4 | Bivariate and multivariate relationships of LVESV reduction}

LVESV reduction was associated with baseline QRS width $(r=0.257$ $p=.002)$, QRS narrowing $(r=0.396, p<.001)$ and CSDI $(r=0.357$, $p<.001)$ from electrocardiographic parameters and NT-proBNP $(r=-0.213, p=.022)$ from laboratory parameters in bivariate analysis. A scatter plot diagram of the relationship between LV lead sensing delay and LVESV reduction is shown in Figure 1. Hemoglobin and creatinine levels were not found to be associated with LVESV reduction in bivariate analysis ( $p>.05$ for all). In logistic regression analysis, CSDI was found to be only independent parameter for predicting significant LVESV reduction (Beta $=0.318, p<.001$ ). Factors used in logistic regression analysis were shown in Table 4.

\subsection{Association of LV lead sensing delay with LVEF increase and QRS narrowing}

CSDI was found to be significantly associated with LVEF increase $(r=0.244, p=.004)$ in bivariate analysis. Scatter plot diagram of the relationship of CSDI with LVEF increase is shown in Figure 2. CSDI was also found to be significantly associated with QRS narrowing $(r=0.178, p=.046)$ in bivariate analysis.

\subsection{ROC curve analysis to determine predictive value of $L V$ lead sensing delay for significant LVESV reduction}

The cutoff value of CSDI obtained by ROC curve analysis was 0.335 for prediction of significant LVESV reduction (sensitivity: 60.0\%, specificity: $59.7 \%)$. The area under the curve (AUC) was $0.685(p<.001)$ (Figure 3).

\section{4 | DISCUSSION}

In our study, we aimed to demonstrate the relationship between echocardiographic, electrocardiographic response to CRT and CSDI. The main findings of the present study are that: (i) CSDI is the only independent predictor for significant $(\geq 15 \%)$ reduction in LVESV; (ii) CSDI was found to be significantly associated with LVEF increase and QRS narrowing after CRT treatment.

CRT treatment has well-established benefits in ventricular remodeling, symptom improvement, exercise tolerance, reducing heart failure hospitalizations and overall mortality (Abraham et al., 2002; Bristow et al., 2004; Cleland et al., 2005). However, 30\%-50\% of patients with indication to CRT treatment do not benefit from this treatment. Predictors of response continues to be an actual focus for clinical research. Clinical investigations have focused on patient selection to reduce the numbers of nonresponders. Appropriate patient selection is a key factor for CRT response. But, it is not clear what the main characteristics of responders except of QRS width longer than $150 \mathrm{~ms}$ and LBBB (Kaszala \& Ellenbogen, 2010). In addition, better patient selection is not enough to reduce non-responders. Because preimplantation factors are not the only predictors of CRT response. Factors associated with implantation and postimplantation period are also as important as preimplantation factors. Different pacing

\begin{tabular}{|lccl|} 
& $\begin{array}{l}\text { Nonresponders (LVESV } \\
\text { reduction }<15 \%) \\
n=67\end{array}$ & $\begin{array}{l}\text { Responders (LVESV } \\
\text { reduction } \geq 15 \%) \\
n=70\end{array}$ & \multicolumn{1}{l}{} \\
\hline Hemoglobin (g/dl) & $12.4 \pm 1.7$ & $13.1 \pm 1.9$ & .028 \\
\hline BUN (mg/dl) & $56.2 \pm 35.6$ & $46.4 \pm 22.7$ & .057 \\
\hline Creatinine $(\mathrm{mg} / \mathrm{dl})$ & $1.47 \pm 1.44$ & $1.04 \pm 0.35$ & .018 \\
\hline Total cholesterol $(\mathrm{mg} / \mathrm{dl})$ & $175.1 \pm 47.3$ & $175.2 \pm 50.2$ & .989 \\
\hline LDL cholesterol $(\mathrm{mg} / \mathrm{dl})$ & $111.9 \pm 34.3$ & $110.4 \pm 39.9$ & .814 \\
\hline HDL cholesterol $(\mathrm{mg} / \mathrm{dl})$ & $38.9 \pm 13.9$ & $40.2 \pm 14.5$ & .591 \\
\hline Triglycerides $(\mathrm{mg} / \mathrm{dl})$ & $166.2 \pm 164.3$ & $191.5 \pm 139.8$ & .346 \\
\hline NT-proBNP $(\mathrm{pg} / \mathrm{ml})$ & $743 \pm 759$ & $450 \pm 481$ & .014 \\
\hline Uric acid $(\mathrm{mg} / \mathrm{dl})$ & $7.1 \pm 2.0$ & $7.0 \pm 2.3$ & .709 \\
\hline
\end{tabular}

TABLE 2 Comparison of laboratory parameters

Bold numbers are statistically significant. BUN, blood urea nitrogen; LDL, low-density lipoprotein; HDL, high-density lipoprotein. 
TABLE 3 Comparison of the baseline electrocardiographic and echocardiographic features of the study population

FIGURE 1 Scatter plot diagram of the relationship between coronary sinus lead delay index and left ventricle end-systolic volume reduction

\begin{tabular}{|c|c|c|c|}
\hline Variable & $\begin{array}{l}\text { Nonresponders (LVESV } \\
\text { reduction < 15\%) } \\
n=67\end{array}$ & $\begin{array}{l}\text { Responders (LVESV } \\
\text { reduction } \geq 15 \% \text { ) } \\
n=70\end{array}$ & $p$ \\
\hline Baseline QRS width (ms) & $139.3 \pm 16.8$ & $147.8 \pm 17.2$ & .004 \\
\hline QRS narrowing (ms) & $14.0 \pm 20.6$ & $28.1 \pm 23.0$ & $<.001$ \\
\hline CSDI & $0.32 \pm 0.11$ & $0.41 \pm 0.15$ & $<.001$ \\
\hline $\begin{array}{l}\text { LV end-diastolic diameter } \\
(\mathrm{mm})\end{array}$ & $58.3 \pm 13.3$ & $61.2 \pm 10.7$ & .164 \\
\hline $\begin{array}{l}\text { LV end-systolic diameter } \\
\text { (mm) }\end{array}$ & $51.5 \pm 13.5$ & $53.5 \pm 9.9$ & .343 \\
\hline Baseline LVEF (\%) & $25.7 \pm 6.3$ & $26.4 \pm 5.4$ & .521 \\
\hline Baseline LVESV (ml) & $171.5 \pm 48.1$ & $173.0 \pm 44.4$ & .849 \\
\hline Baseline LVEDV (ml) & $227.7 \pm 48.2$ & $232.2 \pm 44.2$ & .574 \\
\hline LVESV reduction (\%) & $9.7 \pm 5.5$ & $20.0 \pm 3.0$ & $<.001$ \\
\hline LVEF increase (\%) & $3.22 \pm 4.33$ & $6.77 \pm 5.05$ & $<.001$ \\
\hline $\begin{array}{l}\text { M-mode septal-posterior } \\
\text { delay (ms) (intraventricular } \\
\text { dyssynchrony) }\end{array}$ & $132.8 \pm 71.0$ & $139.9 \pm 79.1$ & .578 \\
\hline $\begin{array}{l}\text { TDI septal-lateral delay } \\
\text { (ms) (intraventricular } \\
\text { dyssynchrony) }\end{array}$ & $41.0 \pm 29.6$ & $41.0 \pm 22.5$ & .997 \\
\hline
\end{tabular}

Bold numbers are statistically significant. LV, left ventricle; LVEF, left ventricle ejection fraction; LVESV, left ventricle end-systolic volume; LVEDV, left ventricle end-diastolic volume; CSDI, coronary sinus lead delay index.

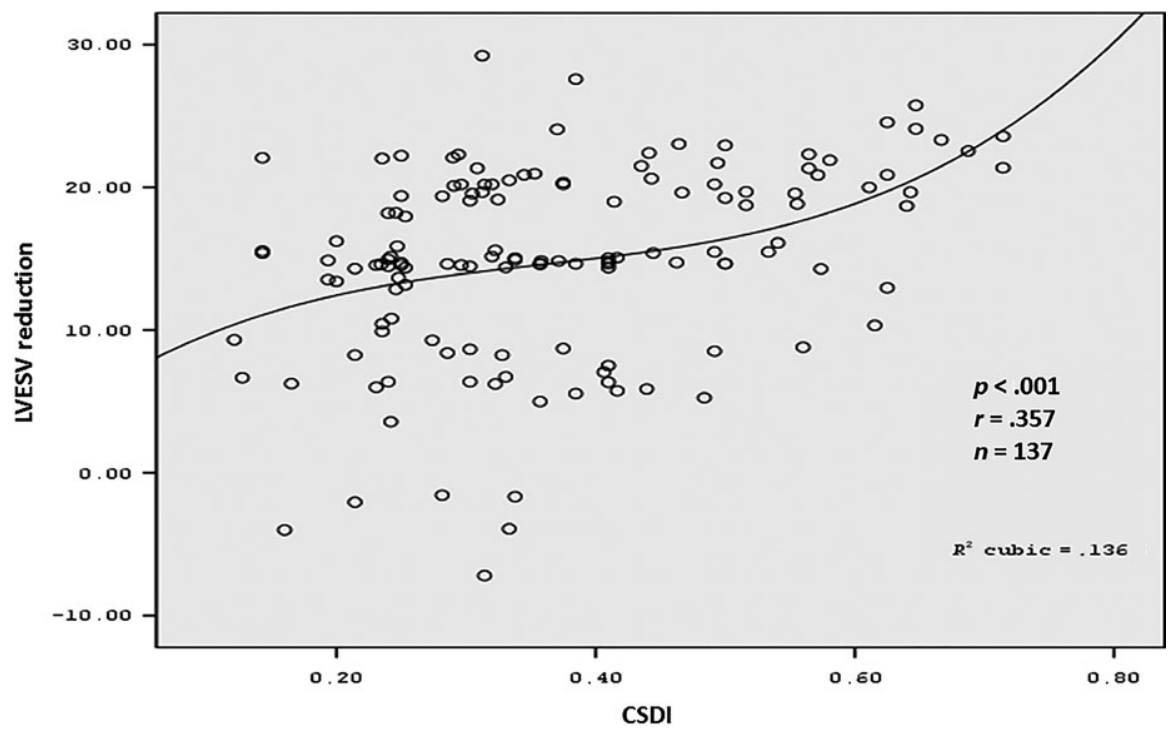

TAB LE 4 Multivariate logistic regression analyses to determine the independent predictors of LVESV reduction

\begin{tabular}{lcc} 
Variable & Beta & $p$ \\
\hline Baseline QRS width (ms) & 0.118 & .286 \\
QRS narrowing (ms) & 0.215 & .062 \\
CSDI & 0.318 & $<.001$ \\
NT-proBNP (pg/ml) & -0.134 & .126 \\
\hline
\end{tabular}

Bold numbers are statistically significant. CSDI, coronary sinus lead delay index. algorithms to reduce intraventricular, interventricular, and atrioventricular dyssynchrony is a continuous field of research in postimplantation period. Regarding implantation factors, position of CS lead and its proximity to the latest activated LV area was found to be a very important factor for CRT response (Liang et al., 2015; Rad et al., 2014).

QRS duration is the major determinative of international guideline recommendations. It is well-known that patients with short QRS durations do not get any benefit from CRT treatment. However, QRS duration alone is not closely associated with pattern or degree of LV activation delay. Because the QRS configuration is a surface depiction 

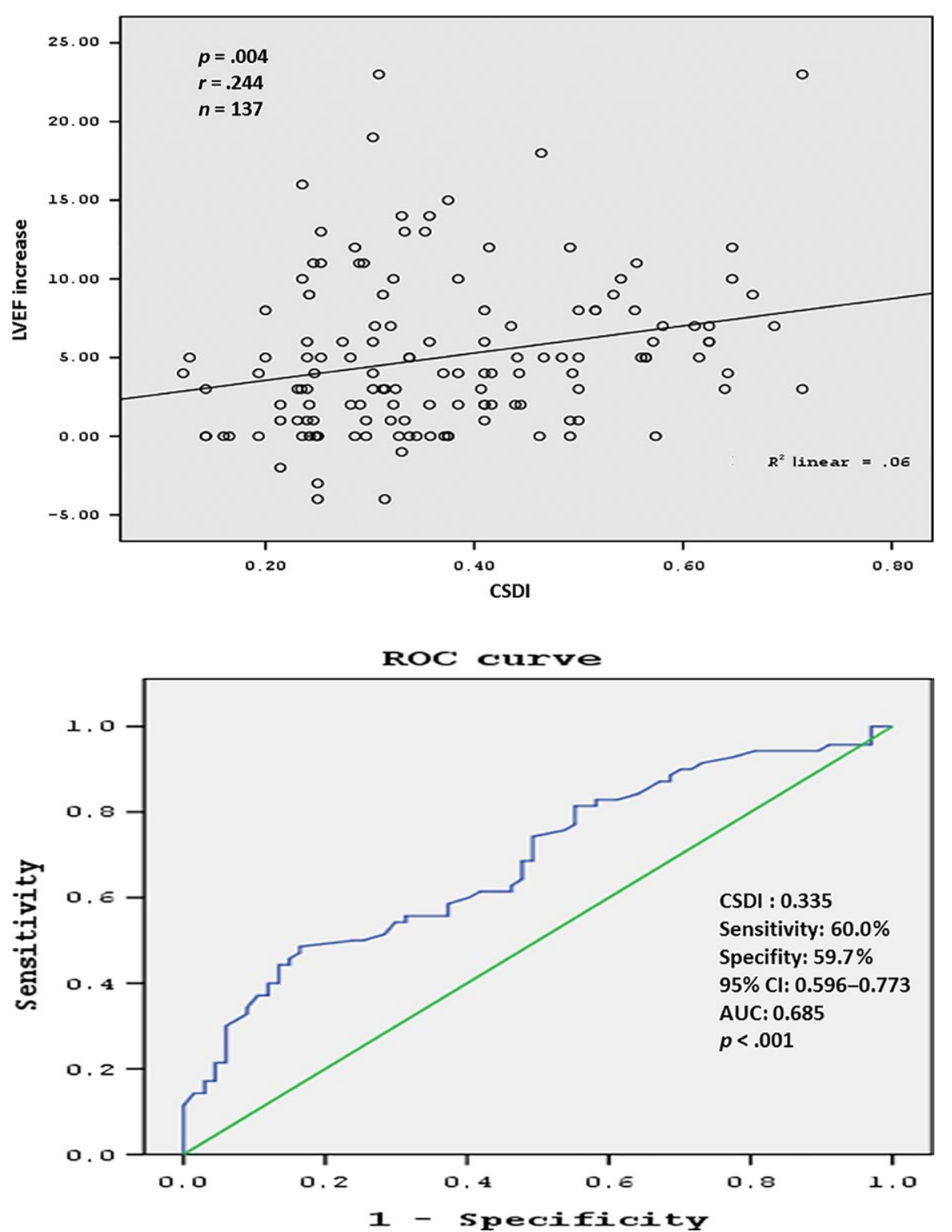

FIGURE 2 Scatter plot diagram of the relationship of coronary sinus lead delay index with left ventricle ejection fraction increase
FIGURE 3 ROC curve analysis to determine predictive value of coronary sinus lead delay index for significant left ventricle end-systolic volume reduction of biventricular activation and may not specifically be a sign of posterolateral, lateral LV activation delay (Varma, 2009). Baseline QRS duration has also been shown to correlate poorly with clinical and echocardiographic outcomes in patients receiving CRT (Mollema, Bleeker, Van Der Wall, Schalij, \& Bax, 2007). In our study, we also found QRS width to be associated with significant reduction in LVESV. But, QRS width was not found to be independent parameter for predicting significant reduction in LVESV in multivariate logistic regression analysis. Likewise, no echocardiographic intraventricular (septum-posterior and septum-lateral delay) dyssynchrony parameters were found to be associated with significant LVESV reduction in our study.

It is very hard to choose the optimal location of the pacing site among available CS branches. The CS lead used to be conventionally positioned in a lateral or posterolateral branches of CS (Butter et al., 2001). Previous studies about reducing non responders had focused on anatomical positions for CS lead placement and did not consider on electrophysiological characteristics of the pacing site. However, anatomic approach is not enough to select the most favorable CS region. Because there is a wide patient to patient variation in both CS anatomy and the anatomic location of the latest activated, LV area with the longest electrical and mechanical activation delay (Rad et al., 2015; Zanon et al., 2014). Before the CRT implantation procedure, echocardiographic approach has been proposed to be used to determine the latest activated LV site (Khan et al., 2012; Ypenburg et al., 2008). But echocardiographic approach has limited accuracy to detect latest activated site and echocardiographic and electrocardiographic activation delays are not always closely correlated. Recently, a new approach, the EAM-guided lead placement was defined by Liang et al. (2012a,b), Del Greco et al. (2012). In the EAM-guided lead placement approach, CS lead is used as a bipolar sensing electrode to calculate the electrophysiologic delay of different CS branches and it is located to the region with longest electrophysiologic delay (Del Greco et al., 2012; Liang et al., 2012b). Coronary venous EAM is easy to execute intraprocedurally using CS lead as a bipolar sensing electrode. There is increasing evidence to suggest that EAM-guided CS lead positioning in the region of latest activation provides superior acute and 
long-term outcome for CRT (Becker et al., 2007; Gold et al., 2011; Khan et al., 2012; Saba et al., 2013; Singh et al., 2006; Ypenburg et al., 2008; Zanon et al., 2014). In the present observational study, we placed the CS lead with conventional anatomical approach, but we measured CS lead sensing delay with EAM using the CS pacing lead as a bipolar electrode. In the study of Liang et al. (2015), they used the delay from the QRS onset to the peak of the CS lead activation wave, but we used the delay from the QRS onset to the beginning of the CS lead activation wave. So, the mean delays in our study was lower than the other studies. In our study, CSDI was the only independent predictor for significant reduction in LVESV and was also found to be significantly associated with significant LVEF increase and QRS narrowing after CRT treatment.

Identifying patients early after device implantation at risk of becoming nonresponders could be advantageous because closer patient follow-up could improve outcomes (Singh et al., 2006).

In addition, CSDI can also be thought to be used in patients with previously implanted CRT devices. CS lead is a bipolar lead and can pace from tip or ring. In patients with previously implanted CRT devices, CS lead can be programmed to pace from the place with higher CSDI (tip or ring). With this approach, there may be a postimplantation optimization of LV pacing site.

We divided coronary sinus lead delay by QRS width in this study. Because in patients with higher QRS duration, CS lead placement with longer coronary sinus lead delays can be thought to be needed in order to minimize posterolateral LV activation delay. Singh et al. (2006) and Roubicek et al. (2015) also divided coronary sinus lead delay by QRS width in their study. They used the delay from the QRS onset to the peak of the CS lead activation wave, but we used the delay from the QRS onset to the beginning of the CS lead activation wave. So, the mean ratios (we named as CSDI, Roubicek et al. (2015) named as QLV ratio) in our study was lower than the other studies.

Previous studies reported female gender, nonischemic cardiomyopathy, LA volume, LV end diastolic volume and QRS $\geq 150 \mathrm{msec}$ to be predictors of echocardiographic CRT response (Goldenberg et al., 2011). LA volume was not analyzed for CRT response prediction in our study. We did not found female gender, nonischemic cardiomyopathy, LV end diastolic volume and QRS $\geq 150 \mathrm{msec}$ as independent predictors of response in our study.

There were some limitations in our study. As a single-center study, our patient cohort might be different from that in other centers. The sample size is relatively small and our results need to be confirmed in future large multicenter prospective trials. Because of the observational nature of our study, we did not use control and EAM-guided CRT groups. So we did not have the chance to make comparisons between the two groups. Patients who have scar tissue in the region of the LV pacing site generally fails to show favorable response to CRT (Adelstein et al., 2011; Bose et al., 2014; Cochet et al., 2013) However, assessment of scar tissue at the pacing site was not performed in the current study. Most studies so far have used LVESV change at 6 months as an endpoint. However, in the current study echocardiographic CRT response has been defined as $\geq 15 \%$ reduction in LVESV at 3 months. In this article, follow-up time for prognostic measurements was shorter than previous studies. However, prognostic follow-up is still going on.

CSDI was found to be significantly associated with LVESV reduction, LVEF increase and QRS narrowing after CRT treatment. We suggest that CSDI may be used as a marker to predict the favorable response to CRT. It may be useful to integrate CSDI to CRT implantation procedure in order to minimize nonresponders. In patients with previously implanted CRT devices, CS lead can be programmed to pace from the place with higher CSDI (tip or ring). Larger and long-term studies are needed to determine the relationship between CSDI and prognostic parameters after CRT treatment.

\section{REFERENCES}

Abraham, W. T., Fisher, W. G., Smith, A. L., Delurgio, D. B., Leon, A. R., Loh, E., ... Messenger, J. (2002). Cardiac-resynchronization in chronic heart failure. New England Journal of Medicine, 346, 1845-1853.

Adelstein, E. C., Tanaka, H., Soman, P., Miske, G., Haberman, S. C., Saba, S. F., ... Gorcsan 3rd, J. (2011). Impact of scar burden by single-photon emission computed tomography myocardial perfusion imaging on patient outcomes following cardiac resynchronization therapy. European Heart Journal, 32, 93-103.

Becker, M., Kramann, R., Franke, A., Breithardt, O. A., Heussen, N., Knackstedt, C., ... Hoffmann, R. (2007). Impact of left ventricular lead position in cardiac resynchronization therapy on left ventricular remodelling. A circumferential strain analysis based on 2D echocardiography. European Heart Journal, 28, 1211-1220.

Bose, A., Kandala, J., Upadhyay, G. A., Riedl, L., Ahmado, I., Padmanabhan, R., ... Singh, J. P. (2014). Impact of myocardial viability and left ventricular lead location on clinical outcome in cardiac resynchronization therapy recipients with ischemic cardiomyopathy. Journal of Cardiovascular Electrophysiology, 25, 507-513.

Bristow, M. R., Saxon, L. A., Boehmer, J., Krueger, S., Kass, DA., De Marco, T., ... Feldman, AM. (2004). Cardiac-resynchronization therapy with or without an implantable defibrillator in advanced chronic heart failure. New England Journal of Medicine, 350, 2140-2150.

Butter, C., Auricchio, A., Stellbrink, C., Fleck, E., Ding, J., Yu, Y., ... Spinelli, J. (2001). Effect of resynchronization therapy stimulation site on the systolic function of heart failure patients. Circulation, 104, 3026-3029.

Cleland, J. G., Daubert, J. C., Erdmann, E., Freemantle, N., Gras, D., Kappenberger, L., \& Tavazzi, L. (2005). The effect of cardiac resynchronization on morbidity and mortality in heart failure. New England Journal of Medicine, 352, 1539-1549.

Cochet, H., Denis, A., Ploux, S., Lumens, J., Amraoui, S., Derval, N., ... Bordachar, P. (2013). Pre- and intra-procedural predictors of reverse remodeling after cardiac resynchronization therapy: An MRI study. Journal of Cardiovascular Electrophysiology, 24, 682-691.

Daubert, J. C., Saxon, L., Adamson, P. B., Auricchio, A., Berger, R. D., Beshai, J. F., ... Zamorano, J. L. (2012). 2012 EHRA/HRS expert consensus statement on cardiac resynchronization therapy in heart failure: Implant and follow-up recommendations and management. Heart Rhythm: the Official Journal of the Heart Rhythm Society, 9, 1524-1576.

Del Greco, M., Marini, M., \& Bonmassari, R. (2012). Implantation of a biventricular implantable cardioverter-defibrillator guided by an electroanatomic mapping system. Europace, 14, 107-111.

Gold, M. R., Birgersdotter-Green, U., Singh, J. P., Ellenbogen, K. A., Yu, Y., Meyer, T. E., ... Tchou, P. J. (2011). The relationship between ventricular electrical delay and left ventricular remodelling with cardiac resynchronization therapy. European Heart Journal, 32, 2516-2524.

Goldenberg, I., Moss, A. J., Hall, W. J., Foster, E., Goldberger, J. J., Santucci, P., ... Klein, H. (2011). Predictors of response to cardiac resynchronization therapy in the Multicenter Automatic Defibrillator Implantation 
Trial with Cardiac Resynchronization Therapy (MADIT-CRT). Circulation, 124(14), 1527-1536.

Kaszala, K., \& Ellenbogen, K. A. (2010). When right may not be right: Right bundle-branch block and response to cardiac resynchronization therapy. Circulation, 122, 1999-2001.

Khan, F. Z., Virdee, M. S., Palmer, C. R., Pugh, P. J., O'Halloran, D., Elsik, M., ... Dutka, D. P. (2012). Targeted left ventricular lead placement to guide cardiac resynchronization therapy: The TARGET study: Arandomized, controlled trial. Journal of the American College of Cardiology, 59, 1509-1518.

Kydd, A. C., Khan, F. Z., Watson, W. D., Pugh, P. J., Virdee, M. S., \& Dutka, D. P. (2014). Prognostic benefit of optimum left ventricular lead position in cardiac resynchronization therapy: Follow-up of the TARGET Study Cohort (Targeted Left Ventricular Lead Placement to guide Cardiac Resynchronization Therapy). JACC Heart Failure, 2, 205-212.

Liang, Y. C., Yu, H. B., Li, S. B., Yang, G. T., Wang, Z. L., \& Han, Y. L. (2012a). Implantation of left ventricular lead guided by electrical mapping in a heart failure patient receiving cardiac resynchronization therapy. Chinese Journal of Cardiac Arrhythmias, 16, 21-22.

Liang, Y. C., Yu, H. B., Sun, Y., Jin, Z. Q., Xu, G. Q., Fu, L. J., ... Han, Y. L. (2012b). The feasibility and curative effect of cardiac resynchronization therapy by targeted left ventricular lead placement to the latest ventricular electrical activating site mapped in the CS branches. Chinese Journal of Cardiac Pacing Electrophysiology, 6, 196-200.

Liang, Y. C., Yu, H. B., Zhou, W. W., Xu, G. Q., Sun, Y., Liu, R., ... Han, Y. L. (2015). Left ventricular lead placement targeted at the latest activated site guided by electrophysiological mapping in coronary sinus branches improves response to cardiac resynchronization therapy. Journal of Cardiovascular Electrophysiology, 26, 1333-1339.

Mollema, S. A., Bleeker, G. B., Van Der Wall, E. E., Schalij, M. J., \& Bax, J. J. (2007). Usefulness of QRS duration to predict response to cardiac resynchronization therapy in patients with end-stage heart failure. American Journal of Cardiology, 100, 1665-1670.

Rad, M. M., Blaauw, Y., Dinh, T., Pison, L., Crijns, H. J., Prinzen, F. W., ... Vernooy, K. (2014). Different regions of latest electrical activation during left bundle-branch block and right ventricular pacing in cardiac resynchronization therapy patients determined by coronary venous electroanatomic mapping. European Journal of Heart Failure, 16, 1214-1222.

Rad, M. M., Blaauw, Y., Dinh, T., Pison, L., Crijns, H. J., Prinzen, F. W., ... Vernooy, K. (2015). Left ventricular lead placement in the latest activated region guided by coronary venous electroanatomic mapping. Europace, 17, 84-93.

Rickard, J. (2012). Cardiac resynchronization therapy. In B. P. Griffin (Ed.), Manual of cardiovascular medicine (pp. 920-930), 4th edn. Philadelphia, USA: Wolters Kluwer.
Roubicek, T., Wichterle, D., Kucera, P., Nedbal, P., Kupec, J., Sedlakova, J., ... Polasek, R. (2015). Left ventricular lead electrical delay is a predictor of mortality in patients with cardiac resynchronization therapy. Circulation: Arrhythmia and Electrophysiology, 8(5), 1113-1121.

Saba, S., Marek, J., Schwartzman, D., Jain, S., Adelstein, E., White, P., ... Gorcsan 3rd, J. (2013). Echocardiography-guided left ventricular lead placement for cardiac resynchronization therapy: Results of the Speckle Tracking Assisted Resynchronization Therapy for Electrode Region trial. Circulation: Heart Failure, 6, 427-434.

Sahn, D. J., De Maria, A., Kisslo, J., \& Weyman, A. (1978). For The Committee on M-Mode Standardization of the American Society of Echocardiography, Recommendations regarding quantitation in $\mathrm{M}$ mode echocardiography: Results of a survey of echocardiographic measurements. Circulation, 58, 1072-1083.

Schiller, N. B. (1991). Two-dimensional echocardiographic determination of left ventricular volume, systolic function, and mass. Summary and discussion of the 1989 recommendations of the American Society of Echocardiography. Circulation, 84, 1280-1287.

Singh, J. P., Fan, D., Heist, E. K., Alabiad, C. R., Taub, C., Reddy, V., ... Mela, T. (2006). Left ventricular lead electrical delay predicts response to cardiac resynchronization therapy. Heart Rhythm: the Official Journal of the Heart Rhythm Society, 3, 1285-1292.

Varma, N. (2009). Cardiac resynchronization therapy and the electrical substrate in heart failure: What does the QRS conceal? Heart Rhythm: the Official Journal of the Heart Rhythm Society, 6, 1059-1062.

Ypenburg, C., van Bommel, R. J., Delgado, V., Mollema, S. A., Bleeker, G. B., Boersma, E., ... Bax, J. J. (2008). Optimal left ventricular lead position predicts reverse remodeling and survival after cardiac resynchronization therapy. Journal of the American College of Cardiology, 52, 1402-1409.

Zanon, F., Baracca, E., Pastore, G., Fraccaro, C., Roncon, L., Aggio, S., ... Prinzen, F. (2014). Determination of the longest intrapatient left ventricular electrical delay may predict acute hemodynamic improvement in cardiac resynchronization therapy patients. Circulation: Arrhythmia and Electrophysiology, 7, 377-383.

How to cite this article: Koç M, Kaypakli O, Gözübüyük G, Sahin D. Coronary sinus lead delay index for optimization of coronary sinus lead placement. Ann Noninvasive Electrocardiol. 2018;23:e12454. https://doi.org/10.1111/anec.12454 